\title{
ASYNCHRONOUS TIME ENCODER BASED QUASI-STATIC MODELLING FOR HUMAN AREA NETWORK IN VANET
}

\author{
Vimal Karthick, R., G.S. Raj, R. Srinivasan, \\ S. Sibi Chakkaravarthy and P. Visu \\ Department of Computer Science and Engineering, \\ Vel Tech Dr. RR and Dr.SR Technical University, Chennai, India
}

Received 2013-12-18; Revised 2014-07-02; Accepted 2014-11-28

\begin{abstract}
Power constraints play a key role in designing Human Area Networks (HANs) for bio authentication vehicle based on driver's identity. To alleviate the power constraints, we advocate a design that uses an asynchronous time encoding mechanisms for representing bio authentication information and the skin surface as the communication channel. Time encoding does not require a clock while allows perfect signal recovery; the communication channel is operated below $1 \mathrm{MHz} \mathrm{We}$ (i) review the fundamental theory behind time encoding and signal recovery, (ii) describe the implementation of a HAN prototype, (iii) describes the implementation of bio authentication for vehicle identity and (iv) present research data obtained from our experimental platform. We demonstrate that the fidelity of the proposed signal representation and transmission scheme is well above the bio medical monitoring requirements even in the case of additive channel-noise and neighbouring channel interference. Consequently, the traditional HAN architecture consisting of clocked A/D converters feeding into digital RF channels can be replaced with a less power demanding time encoding/decoding pair that uses the skin surface as a communications channel. Here we propose a multilayer mathematical model using volume conductor theory for galvanic coupling HAN on a human limb with consideration on the inhomogeneous properties of human tissue. By introducing and checking with quasi-static approximation criteria, Maxwell's equations are decoupled and capacitance effect is included to the governing equation for further improvement. Finally, the accuracy and potential of the model are examined.
\end{abstract}

Keyword:HAN, BAN, Communication, Maxwell Equation, Quasi Static Model, Human Area Network, VANET

\section{INTRODUCTION}

Energy efficiency is of extreme importance in certain biomedical applications such as pulseoximetry, Electrocardiography (ECG) and Electroencephalography (EEG). In contrast, the requirements for accuracy and speed (bandwidth) are rather modest. For example, 8-bit accuracy and $100-500 \mathrm{~Hz}$ bandwidth is typical in ECG and EEG systems (Zimmerman, 1996). The energy Corresponding Author: Vimal Karthick, R., Department of CSE, Vel Tech Dr. RR and Dr.SR Technical University, Chennai, India 
These units enable the deployment of sensor networks for health monitoring often referred to as human, personal or body area networks. In these solutions Analog-to-Digital (A/D) conversion and wireless digital transmission is carried out by the sensor nodes using Radio-Frequency (RF) channels via tiny antennas. Power dissipation due to both $\mathrm{A} / \mathrm{D}$ conversion and digital transmission are two major limitations. A/D converters, Plonsey (2000) and the references there in. In typical recent RF applications the amount of energy needed for transmitting one single bit amounts to that of executing about 1000 of 32-bit computations Shinagawa et al. (2003). Intelligent on-sensor signal processing methods have been investigated with the potential to save power (hence extend battery life) by transmitting processed data rather than raw signals Hachisuka et al. (2006). Figure 1 depicts an alternative method for bio authentication that is closely related to a method originally proposed for digital transmission in Personal Area Network (PAN) applications Hachisuka et al. (2009), Malmivuo and Plonsey (1995). As shown, several sensors and the PDA are connected to the human body via insulators.

The transmit power can be reduced by using the skin surface as a short range communication channel. Briefly, since any two distinct points of the human body are interconnected. via capacitive coupling, signals with high enough frequency content can travel between any two points. Whereas a radio transmitting PAN device needs to be operated at frequencies in the $\mathrm{MHz}$-to- $\mathrm{GHz}$ range in order to efficiently transmit bio authentication information, electrostatic coupling reaches the same efficiency by running the devices at much lower frequencies $(0.1$ to $1 \mathrm{MHz})$. Given that the energy consumption of electronic devices increases with frequency, substantial amount of energy can be saved (Hachisuka et al., 2009). Note that, data transmitted through the human body eventually escapes through the feet into the ground, thereby minimizing the chance of intercept and, thus, providing secure communications (Malmivuo and Plonsey, 1995). Instead of using A/D converters that represent bio authentication information in the amplitude domain, we propose to represent the same information in the time domain with Time Encoding Machines (TEMs) (Xu et al., 2009). TEMs can be typically implemented as low-power, nonlinear, asynchronous, analog circuits and are suitable for IC implementation (Gabriel et al., 1996; Wegmueller et al.,
2009). At a remote site signal reconstruction and application-specific evaluations are carried out. The reconstruction algorithm and its implementation in software and/or hardware is referred to as the Time Decoding Machine (TDM) (Xu et al., 2009).

HAN owns the advantages of low power consumption, reduced electromagnetic radiation, lower interference from external electromagnetic noise, increased security and increased spectrum efficiency. In addition, the design of the HAN transceiver can effectively reduce the complexity of the sensors. For wearable sensors and devices on the body, HAN that uses the human tissue as the communication channel is more natural and convenient. In the implementation stage of HAN, two variations have been developed. The capacitive coupling technique is a two-electrode approach Hachisuka et al. (2006) and the galvanic coupling technique employs four electrodes Wegmueller et al. (2007). The capacitive coupling technique has a higher channel gain Hachisuka et al. (2006) and data rate Shinagawa et al. (2003), while the galvanic coupling technique is capable of both on- body and in-body (i.e., implanted) devices Lindsey et al. (1998), Wegmueller et al. (2009).

In this study, we propose a multilayer mathematical model based on the volume conductor theory that considers the inhomogeneous properties of human tissues to some extent, for galvanic coupling HAN on a human limb in section II. By introducing and checking with quasi-static approximation criteria, Maxwell's equations are decoupled and capacitance effect is introduced to the governing equation for improvement. In sections III and IV, in vitro and in vivo experiments are described and the results are compared with our model, respectively.

Section IV gives result discussion and conclusion is made in section $\mathrm{V}$.

\subsection{How Bio-Authentication Applied for Vehicle Identity?}

Bio authentication is applied for vehicle identity to integrate the driver identity with the vehicular unit; each unit identifies the vehicle based on the broadcasting packets from the driver authentication area through the protocol so far called HAN. A prototype of this protocol has been clearly described in the above section. Based on identity the Tamper proof device in the high end vehicles uses to encode those code (ASCII) with the digital certificates provided by the TPD's. 


\subsection{Encoder}

Here the basic encoding procedure is carried by means of HAN prototype. The basic encoder devices the integrity with the driver, based on integrity it uses a symmetric key algorithm to encrypt the values into a nondecryptable HASH (Daisy chaining).

\subsection{Mathematical Model}

Focusing on the galvanic coupling HAN on the upper human arm, which is a convenient site for experiment realization, the model of limb is approximated by a multilayer concentric cylinder. This simplification is analogous to the research of (Fujii et al., 2007; 2008; Wegmueller et al., 2006; Sasamori et al., 2009; Oh et al., 2007). To elevate the integrity of the model,we have included both permittivity's $([1, \ldots, \ldots)$ and conductivities $(\sigma 1, \ldots, \sigma \mathrm{N})$ with the geometry illustrated in Fig. 1, where h depicts the length of the limb and $\mathrm{r} 1, \ldots$, $\mathrm{rN}$ represent the radii of various tissues. Since the galvanic coupling type HAN generally operated at low frequency, the full-wave formulation of Maxwell's equations was not necessary nor easy to solve. Therefore, we could apply the quasi-static approximation to simplify the HAN problem. In accordance with (Plonsey and Heppner, 1967; Plonsey, 2000), we first checked the quasi static criteria (Handa et al., 1997; Zimmerman, 1996; 1995; Hachisuka et al., 2003; Fukomoto et al., 1997) for potentially decoupling Maxwell's Equation 1 to 4:

Neglect Propagation Effects kRmax $>>1$

Neglect Capacitance Effects $\omega \sigma<<1$

Neglect Inductive Effects (kRmax) $2<<1$

Neglect Leakage to outside air $\omega \sigma N>>0$

Rmax is the maximum length corresponding to the overall dimension of human, $\omega$ is the angular frequency, $\omega \sigma$ and $\sigma$ are the permittivity and conductivity of tissues, $\mathrm{k} R \max$ is the permittivity of free space, $\sigma \mathrm{N}$ is the conductivity of the outmost tissue layer and $\mu$ is the permeability of tissues.

\subsection{Implementation of HAN Prototype}

\subsubsection{Experimental Environment}

Our controlled environment for measurements and evaluations is depicted in Fig. 3. Exact error evaluations require the accurate knowledge of the input signals. Since real sensors do not faithfully represent their input, we opted to employ ECG recorded signals instead. As seen, the definition of the input signal $u(t)$, the implementation of the PDA and the TDM and the performance evaluation were carried out in a computational environment.

The TEM, the modulator and the demodulator were implemented using standard discrete-component circuit elements and commercially available ICs. In our experiments we used both skin and a skin substitute (a) wet sponge placed in a metalized plastic bag used for electrostatic discharge storage (Malmivuo and Plonsey, 1995). The main (solid line) and the auxiliary (dashed line) programmable generators (Tektronix AFG 3252) create $u(t)$ shown in Fig. 4 and the interfering signals (crosstalk from other channel and noise), respectively. Any node voltage in the circuits can be measured and the measurements (samples with given sampling frequency) can be stored by a digital oscilloscope.

Software package (ArbExpress, Tektronix) allows data transfer between the computational environment and the generator and/or oscilloscope using the CSV (comma-separated value) file format. As shown, the samples of the demodulator output serve as input to the reconstruction (PDA and TDM) and the performance evaluation module. The calibration unit evaluates relevant error measures and establishes the accuracy limits of the arrangement. For completeness, we included a brief description of error measures and calibration.

\subsection{Reconstruction Accuracy in the Computational Environment}

In order to emulate a realistic class of sensor generated signals, we selected an ECG signal from the MIT-BIH public arrhythmia database (Sasamori et al., 2009). This database contains a number of ambulatory ECG recordings digitized at 360 samples per second with 11-bit resolution over a $10 \mathrm{mV}$ range. One of the (scaled) signal segments obtained after a 7-th order polynomial interpolation. Due to measurement constraints, we employed a periodic input in our experimental platform. A periodic band limited waveform $\mathrm{u}(\mathrm{t})$ was generated by expanding a segment of length $2.5 \mathrm{~s}$ of the signal into Fourier-series. The fast FFT algorithm computed 750 Fourier coefficients. Measured results of $\mathrm{u}(\mathrm{t})$ so obtained and employed in the subsequent experiments. Therefore the bandwidth of $\mathrm{u}(\mathrm{t})$ amounts to $=750 \times(2 \pi / 2.5)=$ $2 \pi \times 300 \mathrm{rad} / \mathrm{s}$. As shown, $\mathrm{c}=0.3$ is an appropriate amplitude bound on the emulated ECG signal. With 
TEM parameters $\delta=0.17, \mathrm{~b}=0.6$ and $\kappa=0.001 \mathrm{~s}$, the condition in (3) is satisfied with $\kappa \delta=0.402(\mathrm{~b}-\mathrm{c}) \pi /$. For $\mathrm{u}(\mathrm{t})$ in Fig. 4 the numerical calculation of 4975 Tk's was carried out by solving (1) recursively. Using these values we determined the TEM signals $y(t)$ and $z(t)$ for isualization purposes as shown in Fig. 5. Setting the sampling rate of the TDM to $\mathrm{S}=\pi /(4)=3.472 \mathrm{~ms}$, the recovery error evaluated according to (A-4).

\subsection{Tamper Proof Circuit (TPD Circuit)}

Figure 6 shows the circuit implementation of the TEM in Fig. 2 using resistors and capacitors with $2 \%$ tolerance, LF256 opamps and diodes. The middle opamp with the $47 \mathrm{nF}$ capacitor and $28 \mathrm{k}$ resistor implements the integrator. The right most opamp with resistors $1,56.3,11.5 \mathrm{k}$.

The diodes force the trigger output to take voltages around $\pm 0.6 \mathrm{~V}$ independent of the $\pm 9 \mathrm{~V}$ power supply voltages of the opamps (not shown). In this way, the TEM can be operated properly by batteries for relatively long time. The adder implemented by the leftmost opamp and the $100 \mathrm{k}$ resistors allow measuring the sum of $\mathrm{u}(\mathrm{t})$ and the Schmitt-trigger output for test purposes.

With the resistance and capacitance values shown, we set the same TEM parameter values (b, $\delta$ and $\kappa$ ) as those in the example of Section III-B. With zero input, the self oscillation frequency.

\subsection{Performance Evaluation}

In what follows we reconstruct the samples of a segment of $\mathrm{u}(\mathrm{t})$ in Fig. 6 with and without noise or interference due to a neighbouring channel. The same parameters for the input-signal bandwidth and the sampling frequency of the reconstructed signal are used as in section III-B. The Tk's were determined by the algorithm implementing the functionality depicted in Fig. 6 with appropriate levels for $q 1$ and $q 2$. The evaluation is based on measuring the Root-MeanSquare (RMS) error between the reconstructed samples $\mathrm{u}[\mathrm{kS}]$ and the samples $\mathrm{u}(\mathrm{kS})$ as described in Appendix VI-B.

\subsection{Evaluation without Interference and Noise}

In this section the reconstruction of the signal $\mathrm{u}(\mathrm{t})$ is considered without sources of noise or interference. With levels $\mathrm{q} 1=2.5$ and $\mathrm{q} 2=2.7$, the Tk's were digitized by employing several time-measuring frequency values fCLK. First, the number of bits needed to represent each Nk in Fig. 6 was determined. Then, the bit rate was calculated as the ratio of the total number of bits so obtained and the time interval over which the reconstruction was carried out. Figure 2 shows the RMS reconstruction errors and the corresponding bit rate as a function of fCLK.

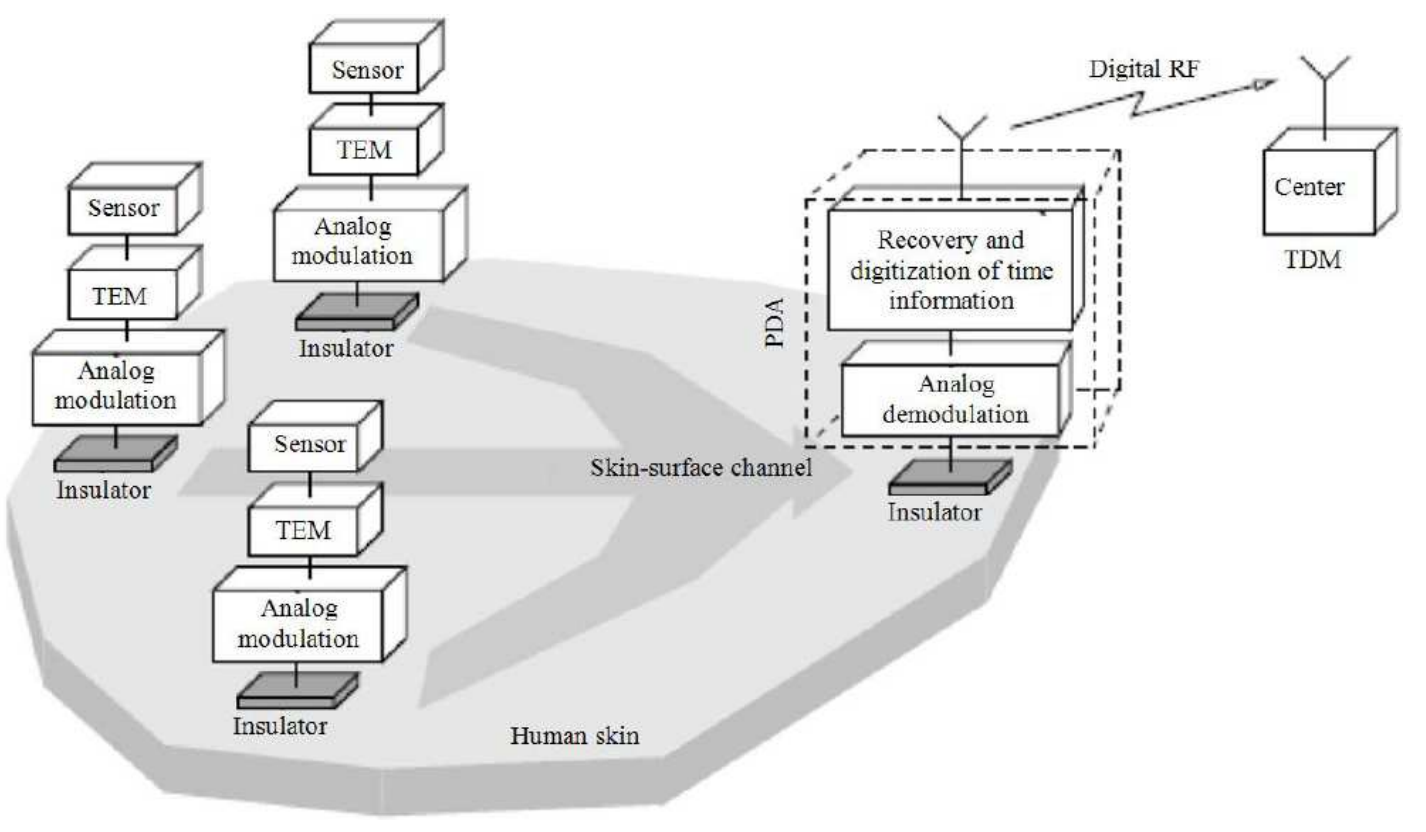

Fig. 1. Basic architecture of human area networking for bio authentication 


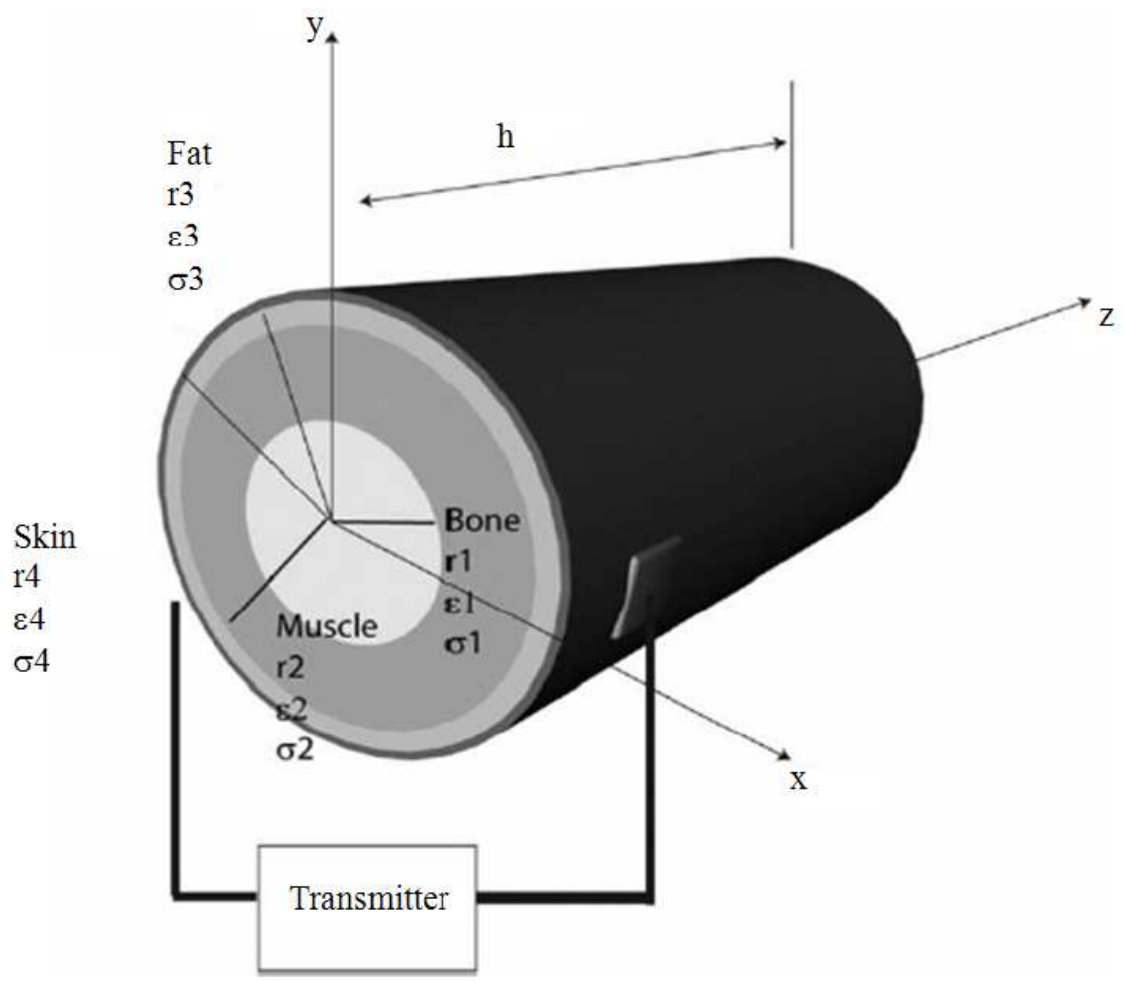

Fig. 2. Representation of HAN limb model

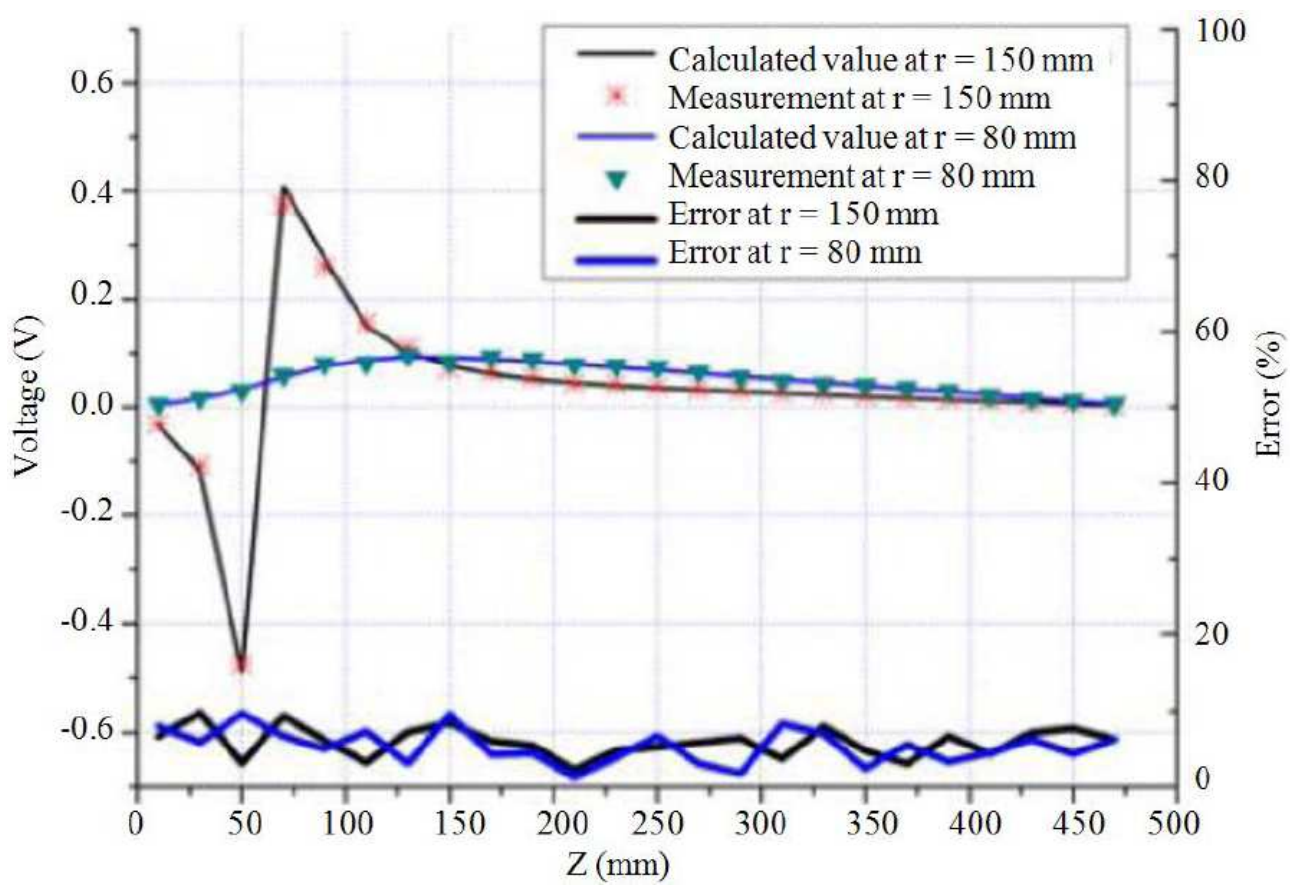

Fig. 3. Comparison of the measured and the calculated results in the z-direction of the phantom 


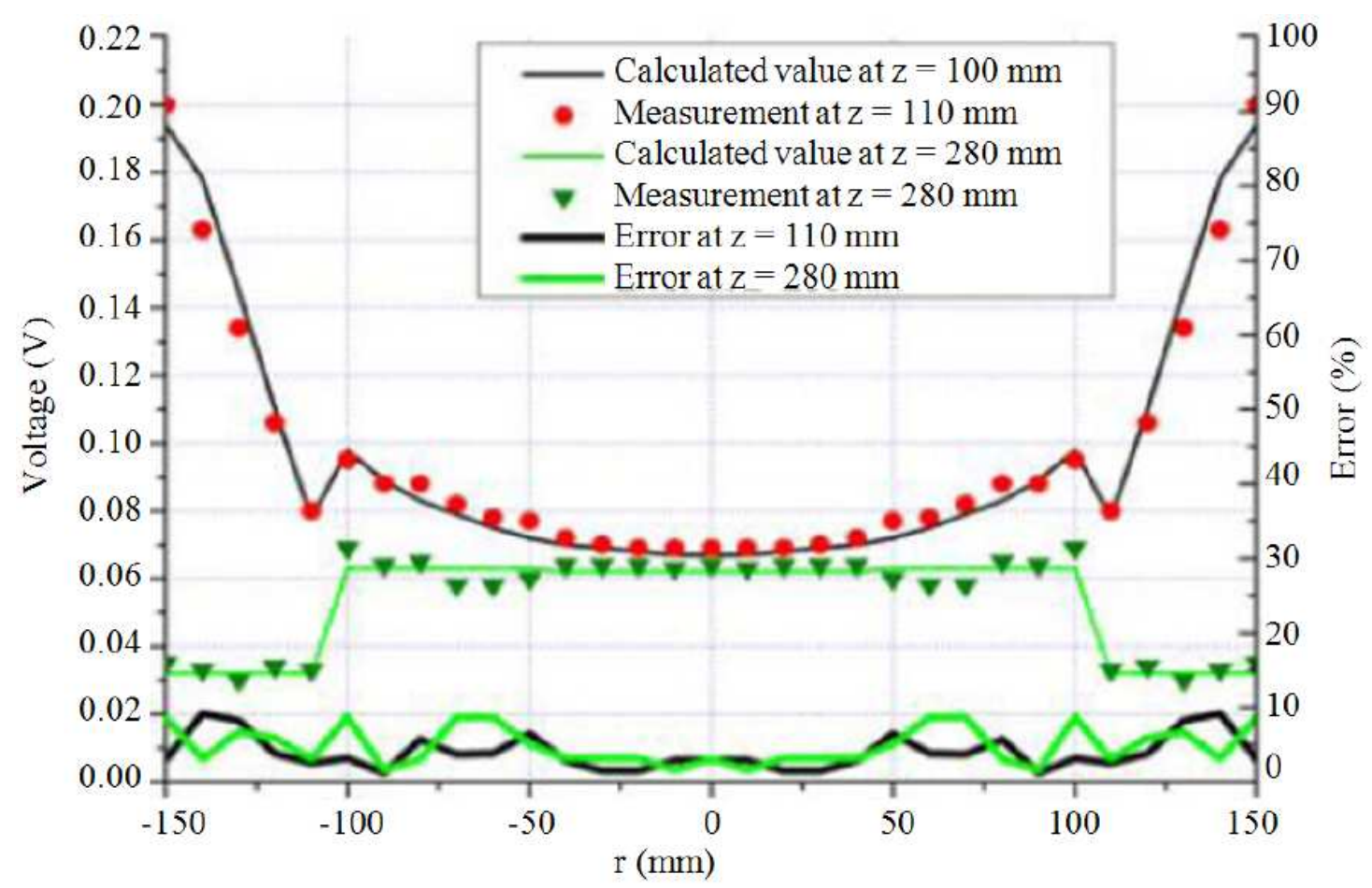

Fig. 4. Comparison of the measured and the calculated results in the z-direction of the phantom

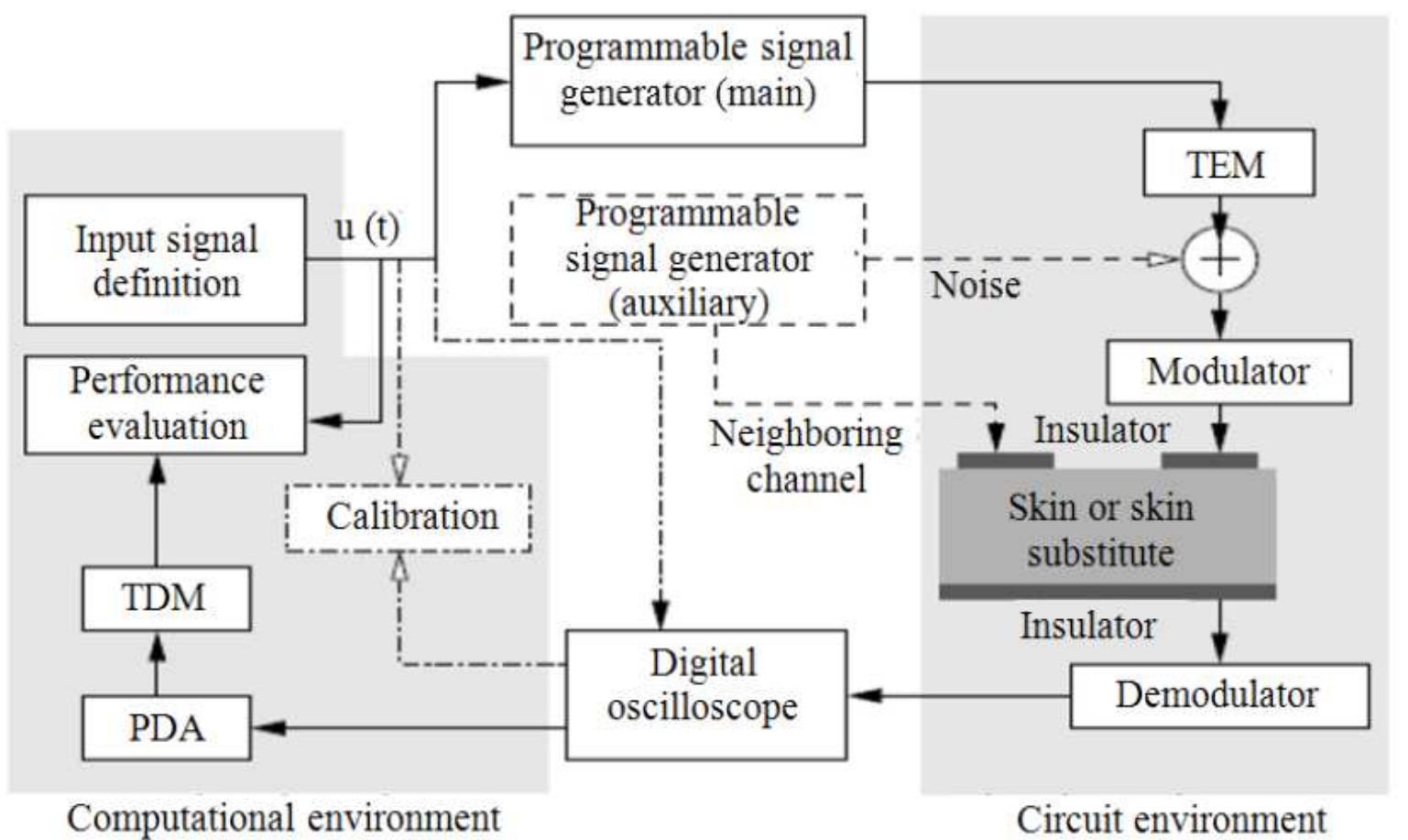

Fig. 5. Basic HAN proto build type 


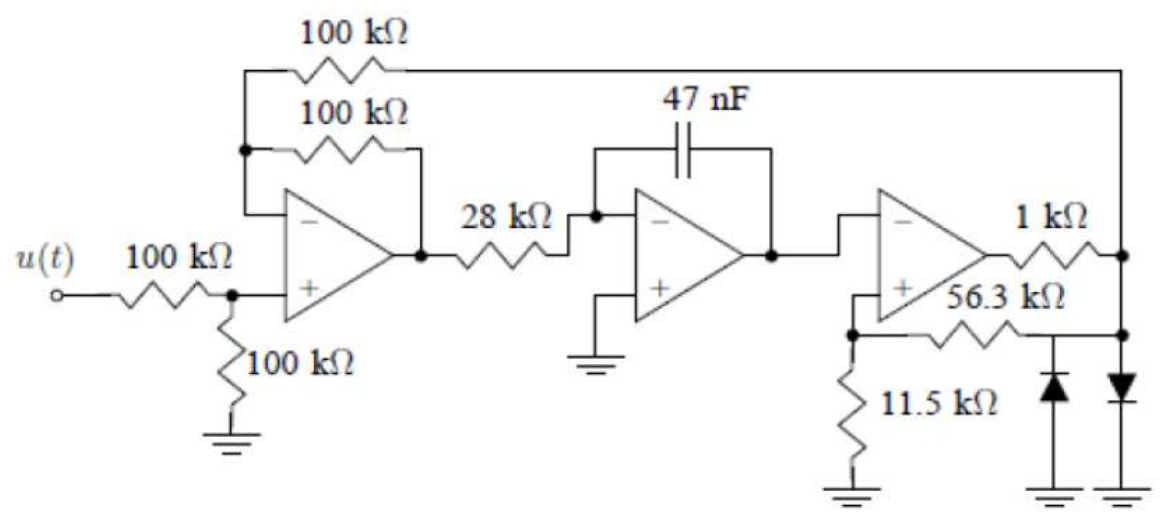

Fig. 6. Encoder slot tamper proof circuit for vehicular modem

As seen, for high enough fCLK values, the accuracy of the reconstruction is limited by circuit imperfections and the skin-surface channel. Figure 2 shows the reconstructed samples with fCLK $=1 \mathrm{GHz}$ on a $1.9 \mathrm{sec}$ time range for $\mathrm{u}(\mathrm{t})$.

\section{CONCLUSION}

We have investigated architecture for Human Area Networks that is based on representing bio authentication information in the time domain and uses the skin surface to transmit this representation to a TPD's in vehicles. We demonstrated that, the performance of such a system is above the requirements adopted in practice. In our experimental environment we used a simple to implement AM/FM modulation/demodulation scheme. Although AM-based schemes are sensitive to interference, while FM values are reverse modulation based schemes which is insensitive to proto build, the evaluation of our prototype has demonstrated a level of performance acceptable in bio authentication applications. We expect to readily improve on our results by using better suited modulation schemes. Although slight errors exist, the comparisons between the measurement and calculation results show that the model is matched with the experiment data not only limited to the surface of the phantom, but also the inside of the various tissues. The experiment also shows the possibility of using galvanic coupling HAN for communicating with implantable devices, although the attenuation is higher than those receiving electrodes situated on the surface. From the model and the experiments, we can surmise the path of the major propagating medium of HAN. The calculated results show that the amount of radiation from the tissue is small and negligible. On the other hand, both the measured and the calculated results show that the gain of HAN is higher than-75 dB, which is the measure gain between the transmitter and the receiver electrodes through air in our experimental setting. Thus, one can conclude that the major propagation path of HAN is the human limb and negligible portion of the propagation over the air exists for operating frequency below $1 \mathrm{MHz}$ In the next step, inclusion of the anisotropic characteristics of the human tissue shall be investigated in order to increase the accuracy of the model. Moreover, extending the model to other parts of the human body and investigation on modulating schemes are also worth studying. Our model provides a high integrity check for each node identities in the intra and inters Vehicle communication.

\section{REFERENCES}

Fujii, K., D. Ishide, M. Takahashi and K. Ito, 2008. Electric field distributions generated by a wearable device using simplified whole human body models. Inform. Med. Technol., 4: 647-654.

Fujii, K., M. Takahashi and K. Ito, 2007. Electric field distributions of wearable devices using the human body as a transmission channel. IEEE Trans. Antennas Propag., 55: 2080-2087. DOI: 10.1109/TAP.2007.900226

Fukomoto, M., M. Shinagawa and T. Sugimura, 1997. Body coupled Fingerring: Wireless wearable keyboard. Proceedings of the ACM SIGCHI Conference on Human Factors in Computing Systems, Mar. 22-27, ACM New York, NY, USA, pp: 147-154. DOI: $10.1145 / 258549.258636$

Gabriel, S., R.W. Lau and C. Gabriel, 1996. The dielectric properties of biological tissues: III. Parametric models for the dielectric spectrum of tissues. Phys. Med. Biol., 41: 2271-2293. DOI: 10.1088/0031-9155/41/11/003 
Hachisuka, K., A. Nakata, T. Takeda, K. Shiba and K. Sasaki et al., 2003. Development of wearable intrabody communication devices. Sens. Actuators A, Phys., 105: 109-115. DOI: 10.1016/S09244247(03)00060-8

Hachisuka, K., A. Nakata, T. Takeda, Y. Terauchi and K. Shiba et al., 2009. Development and performance analysis of an intra-body communication device.

Hachisuka, K., Y. Terauchi, Y. Kishi, K. Sasaki and T. Hirota et al., 2006. Simplified circuit modeling and fabrication of intrabody communication devices. Sens. Actuators A, Phys., 130-131: 322-330. DOI: 10.1016/j.sna.2006.04.044

Handa, T., S. Shoji, S. Ike, S. Takeda and T. Sekiguchi et al., 1997. A very low-power consumption wireless ECG monitoring system using body as a signal transmission medium. Proceedings of the International Conference on Solid State Sensors and Actuators, Jun. 16-19, IEEE Xplore Press, Chicago, IL, pp: 1003-1006. DOI: 10.1109/SENSOR.1997.635344

Lindsey, D.P., E.L. Mckee, M.L. Hull and S.M. Howell, 1998. A new technique for transmission of signals from implantable transducers. IEEE Trans. Biomed. Eng., 45: 614-619. DOI: 10.1109/10.668752

Malmivuo, J. and R. Plonsey, 1995. Bio Electromagnetism. 1st Edn., Oxford Univ. Press, New York.

Oh, J., J. Park, H. Lee and S. Nam, 2007. The electrode structure to reduce channel loss for human body communication using human body as transmission medium. Proceedings of the IEEE Antennas and Propagation Society International Symposium, Jun. 9-15, IEEE Xplore Press, Honolulu, HI, pp: 15171520. DOI: 10.1109/APS.2007.4395795

Plonsey, R. and E.B. Heppner, 1967. Considerations of quasi-stationarity in electrophysiological systems. Bull. Math. Biophys., 29: 657-664. DOI: 10.1007/BF02476917

Plonsey, R., 2000. Volume Conductor Theory. In: The Biomedical Engineering Handbook, Bronzino, J.D., (Ed.)., Boca Raton, FL: CRC Press.
Sasamori, T., M. Takahashi and T. Uno, 2009. Transmission mechanism of wearable device for on body wireless communications. IEEE Trans. Antennas Propag., 57: 936-942. DOI: 10.1109/TAP.2009.2014575

Shinagawa, M., M. Fukamoto, K. Ochiai and H. Kyuragi, 2003. A near-fieldsensing transceiver for intra-body communication based on the electrooptic effect. Instrum. Measur. IEEE Trans., 53: 296-301. DOI: 10.1109/TIM.2004.834064

Wegmueller, M.S., A. Kuhn, J. Froehlich, M. Oberle and N. Felber et al., 2007. An attempt to model the human body as a communication channel. IEEE Trans. Biomed. Eng., 54: 1851-1857. DOI: 10.1109/TBME.2007.893498

Wegmueller, M.S., M. Oberle, N. Kuster and W. Fichtner, 2006. From dielectrical properties of human tissue to intra-body communications. World Congr. Med. Phys. Biomed. Eng., 14: 613-617. DOI: 10.1007/978-3-540-36841-0_141

Wegmueller, M.S., S. Huclova, J. Froehlich, M. Oberle and N. Felber et al., 2009. Galvanic coupling enabling wireless implant communications. IEEE Trans. Instrum. Meas., 58: 2618-2625. DOI: 10.1109/TIM.2009.2015639

Xu, R., H. Zhu and J. Yuan, 2009. Characterization and analysis of intra-body communication channel. Proceedings of the IEEE Antennas and Propagation Society International Symposium, Jun. 1-5, IEEE Xplore Press, Charleston, SC, pp: 1-4. DOI: 10.1109/APS.2009.5172001

Zimmerman, T.G., 1995. Personal Area Networks: Nearfield intrabody communication. IBM Syst. J., 35: 609-617. DOI: $10.1147 /$ sj.353.0609

Zimmerman, T.G., 1996. Personal Area Networks: Nearfield intrabody communication. IBM Syst. J., 35: 609-617. DOI: 10.1147/sj.353.0609 